\title{
Improved finite-source inversion through joint measurements of rotational and translational ground motions: a numerical study
}

\author{
Michael Reinwald ${ }^{1}$, Moritz Bernauer ${ }^{2}$, Heiner Igel ${ }^{2}$, and Stefanie Donner ${ }^{2}$ \\ ${ }^{1}$ Laboratoire d'Imagerie Biomédicale, UPMC, Paris, France \\ ${ }^{2}$ Department of Earth and Environmental Sciences, LMU Munich, Munich, Germany \\ Correspondence to: Michael Reinwald (mchlrnwld@gmail.com)
}

Received: 20 April 2016 - Published in Solid Earth Discuss.: 26 April 2016

Revised: 12 July 2016 - Accepted: 4 October 2016 - Published: 21 October 2016

\begin{abstract}
With the prospects of seismic equipment being able to measure rotational ground motions in a wide frequency and amplitude range in the near future, we engage in the question of how this type of ground motion observation can be used to solve the seismic source inverse problem. In this paper, we focus on the question of whether finite-source inversion can benefit from additional observations of rotational motion. Keeping the overall number of traces constant, we compare observations from a surface seismic network with 44 three-component translational sensors (classic seismometers) with those obtained with 22 sixcomponent sensors (with additional three-component rotational motions). Synthetic seismograms are calculated for known finite-source properties. The corresponding inverse problem is posed in a probabilistic way using the Shannon information content to measure how the observations constrain the seismic source properties. We minimize the influence of the source receiver geometry around the fault by statistically analyzing six-component inversions with a random distribution of receivers. Since our previous results are achieved with a regular spacing of the receivers, we try to answer the question of whether the results are dependent on the spatial distribution of the receivers. The results show that with the six-component subnetworks, kinematic source inversions for source properties (such as rupture velocity, rise time, and slip amplitudes) are not only equally successful (even that would be beneficial because of the substantially reduced logistics installing half the sensors) but also statistically inversions for some source properties are almost always improved. This can be attributed to the fact that the (in particular vertical) gradient information is contained in the additional motion components. We compare these effects for
\end{abstract}

strike-slip and normal-faulting type sources and confirm that the increase in inversion quality for kinematic source parameters is even higher for the normal fault. This indicates that the inversion benefits from the additional information provided by the horizontal rotation rates, i.e., information about the vertical displacement gradient.

\section{Introduction}

The inversion for kinematic finite-source models of earthquakes plays an important role in the research of earthquake dynamics and the general understanding of earthquakes. Finite-source inversion of earthquakes is non-unique due to the infinite-dimensional inverse problem, which we try to solve with a finite number of observations. A discretization of the fault area by subfaults converts this problem to a finite size inversion, but this does not completely remove the inherent non-uniqueness of the problem. Besides that, noisy data, sparse geographical coverage of seismic stations, the non-linearity of the forward problem, and possible unrealistic simplifications in the parametrization of the fault also contribute to the non-uniqueness.

According to classical elasticity theory, if $\boldsymbol{u}(\boldsymbol{x})$ is the displacement of a particle at position $x$ and if deformation is considered to be infinitesimal, the displacement of a particle at position $\boldsymbol{u}(\boldsymbol{x}+\delta \boldsymbol{x})$ can be described with the following equation (Aki and Richards, 2002):

$\boldsymbol{u}(\boldsymbol{x}+\delta \boldsymbol{x})=\boldsymbol{u}(\boldsymbol{x})+\boldsymbol{\epsilon} \cdot \delta \boldsymbol{x}+\boldsymbol{\omega} \times \delta \boldsymbol{x}$.

In Eq. (1), $\epsilon$ is the symmetric strain tensor, therefore containing six instead of nine independent components, and 
$\boldsymbol{\omega}=\left(\frac{1}{2} \nabla \times \boldsymbol{u}\right)$ are the rotations around each of the three translational axes of $\boldsymbol{u}$. Therefore the full description of a displacement around a point $x$ needs three translational, three rotational, and six strain components. The rotation components are mathematically related to the space derivatives of the translational motion components $u_{x}, u_{y}$, and $u_{z}$ :

$$
\left(\begin{array}{l}
\omega_{x} \\
\omega_{y} \\
\omega_{z}
\end{array}\right)=\frac{1}{2}\left(\begin{array}{l}
\partial_{x} \\
\partial_{y} \\
\partial_{z}
\end{array}\right) \times\left(\begin{array}{l}
u_{x} \\
u_{y} \\
u_{z}
\end{array}\right)=\frac{1}{2}\left(\begin{array}{l}
\partial_{y} u_{z}-\partial_{z} u_{y} \\
\partial_{z} u_{x}-\partial_{x} u_{z} \\
\partial_{x} u_{y}-\partial_{y} u_{x}
\end{array}\right) .
$$

Although rotational ground motions are caused by earthquake source processes, measurements thereof had not been carried out until the 1990s (Nigbor, 1994; Takeo, 1998). Nevertheless, the possible benefit was considered decades before - in the first edition of Aki and Richards (2002), which was published in 1980. Even then, the methods that were used mostly consisted of several three-component seismometers and were not able to be used for seismology because of their low sensitivity (Suryanto, 2006). Since then, many scientists commended the benefits of studying rotational ground motions for Earth sciences (Twiss et al., 1993; Spudich et al., 1995; Takeo and Ito, 1997; Igel et al., 2007; Fichtner and Igel, 2009), physics (DeSalvo, 2009; Lantz et al., 2009), and also for engineering applications (Trifunac, 2009). In the last years, new instruments, e.g., ring-laser technology (Schreiber et al., 2009; Velikoseltsev et al., 2012) or adapted gyroscopes (Bernauer et al., 2012), that are capable of measuring rotational motions with a much higher precision have been developed and possibly bring new insight into seismological applications.

With the potential availability of seismometers measuring rotational ground motions, the goal of this study is to test the effect of incorporating rotational ground motion data into finite-source inversions for different fault mechanisms. Following the approach by Bernauer et al. (2014), we employ a Bayesian, i.e., probabilistic, inversion algorithm and compare the inversions with different data types and different earthquake source mechanisms. Our data consist of wave-field seismograms calculated at receiver positions at the Earth's surface by numerical simulations in six components (three translational and three rotational components). In the last decade there have been several studies showing that probabilistic methods are well suited for ill-posed inversion problems of finite-source earthquakes, because they provide efficient methods to identify the drawbacks of regularization techniques such as local minima (Monelli and Mai, 2009; Fichtner and Tkalčić, 2010) and take them into account when inversion results are interpreted.

We directly compare the results with and without incorporation of rotational ground motion data for a pure strike-slip earthquake and for a dip-slip earthquake; i.e., the strike also propagates in vertical direction. Because rotations around the horizontal axes (i.e., $\omega_{x}$ and $\omega_{y}$ in Eq. 2) carry information about vertical gradients, which is not possible to resolve with common arrays on the Earth's surface (Bernauer et al., 2014), we expect to see an even greater benefit for inversions for the dip-slip scenario due to the higher energy in the horizontal rotation components.

To better assess the quality differences we keep the number of seismograms used in the inversion the same for all scenarios. Because using three translational and three rotational ground motion recordings doubles the number of seismograms, we half the number of seismic stations when using the so-called 6C (six-component) data, as compared to only three translational seismograms (3C/three-component data). In the following chapter, we present the Bayesian inversion scheme. Next, we explain the parametrization of the synthetic earthquakes, explain the spatial distribution of the seismic stations, and define the different inversion scenarios. We will then present the inversion results with and without incorporation of the rotational ground motion data for the two different earthquake mechanisms.

\section{Probabilistic inversion scheme}

Although computation time is drastically increased when applying probabilistic inversion schemes, it is able to quantitatively analyze drawbacks such as regularization or falling into local minima in the iterations during the process of minimizing the misfit between model and data. We use the basic ideas of Bayesian inversion for a finite fault developed by Tarantola (2005) and Mosegaard and Tarantola (1995): for each inversion, a certain number of parameters is stored in the vector $\boldsymbol{m}$, given by

$\sigma(\boldsymbol{m})=\kappa \cdot \rho(\boldsymbol{m}) L(\boldsymbol{m})$,

with the normalization constant $\kappa$ and the associated probability density functions (pdf's) of the prior $\rho(\boldsymbol{m})$ and posterior probability $\sigma(\boldsymbol{m})$ on the model parameters $\boldsymbol{m} . L(\boldsymbol{m})$ is defined as the likelihood function, which features information about how well the model explains the data.

$L(\boldsymbol{m})=\exp (-\chi(\boldsymbol{m}))$

In Eq. (4), $\chi(\boldsymbol{m})$ denotes the norm in the data space used to measure the distance between predicted and observational data.

The prior pdf $\rho(\boldsymbol{m})$ is set to be constant in all model parameters within a certain interval of interest. Since all model parameters are independent, the combined prior pdf can be obtained by simply multiplying the probability densities for all parameters. We approximate the posterior pdf for all parameters by applying the Metropolis algorithm (Hastings, 1970) to 1000000 test models. Once the entire model space is sampled, one can illustrate it by marginal density distributions of each of the parameters. The shape of these distributions gives a discrete approximation of the posterior pdf.

To quantify the posterior pdf compared to the prior pdf, i.e., the relative information gain, we use the so-called Shannon's measure of information gain: the relative information 
gain $I(\rho ; \sigma)$ with respect to the posterior pdf $\rho$ and the prior $\operatorname{pdf} \sigma$ is defined in Tarantola (2005) as

$I(\rho ; \sigma)=\int \rho(x) \log _{2}\left[\frac{\rho(x)}{\sigma(x)}\right] \mathrm{d} x$.

Since the base of the logarithm in Eq. (5) is 2, the unit of $I$ is termed a bit. One major advantage of this method is the possible comparison between results of very different experiments by quantification of the inversion quality. For further details on the method we refer to the publications of Bernauer et al. (2014) and Tarantola (2005).

\section{Forward model}

The generation of the databases, in which Green's functions are stored, is done with the program Instaseis (van Driel et al., 2015), based on the parallel spectral-element method AxiSEM (Nissen-Meyer et al., 2014), which allows for nearinstantaneous (on the order of milliseconds) extraction of seismograms. We used the velocity model ak $135 \mathrm{f}$ in our simulations (Kennett et al., 1995).

We illustrate receiver and fault setup in Fig. 1. A total of 44 receivers, marked as gray, and red triangles, are set up around the source with a spacing of $0.4^{\circ}$ latitude and $0.25^{\circ}$ longitude at the Earth's surface to guarantee a good coverage in all directions from the fault. Since we want to decrease the number of receivers by a factor of 2 when incorporating rotational ground motion data to keep the amount of data used in the inversion the same, we will limit our recording to the 22 stations marked with red triangles, when using both data types. The fault trace, illustrated as a black line, has a constant latitude of $0^{\circ}$ and the epicenter of the earthquake is marked with a yellow star. At this point we want to emphasize that, due to the theoretical nature of this study, the geographical location of fault and receivers are in no way related to real data of past earthquakes or geographical areas.

The parametrization of the fault and the rupture itself is used in the same manner as in Bernauer et al. (2014): we break up the fault plane into $8 \times 3=24$ quadratic subfaults. Each subfault is filled by $8 \times 8=64$ moment tensor point sources with a regular spacing of $500 \mathrm{~m}$ between the point sources. The side length of each subfault is therefore about $4 \mathrm{~km}$. We illustrate the fault in a 2-D sketch shown in Fig. 2. All of the moment tensor point sources are illustrated by small black dots, and the subfaults are patterned in a gray/white color scheme for easier distinction of the subfaults. We set the hypocenter - marked as a yellow star - to be exactly between four subfaults. We allow each subfault to slip once, with a delay of the average time of all rupture times of the 64 point sources in the subfault. This is calculated with a homogeneous rupture velocity $c_{\text {rup }}=2700 \mathrm{~m} \mathrm{~s}^{-1}$ across the entire fault and the computed distances of each point source. We compute a library with the seismic response of each subfault by summing over the previously computed

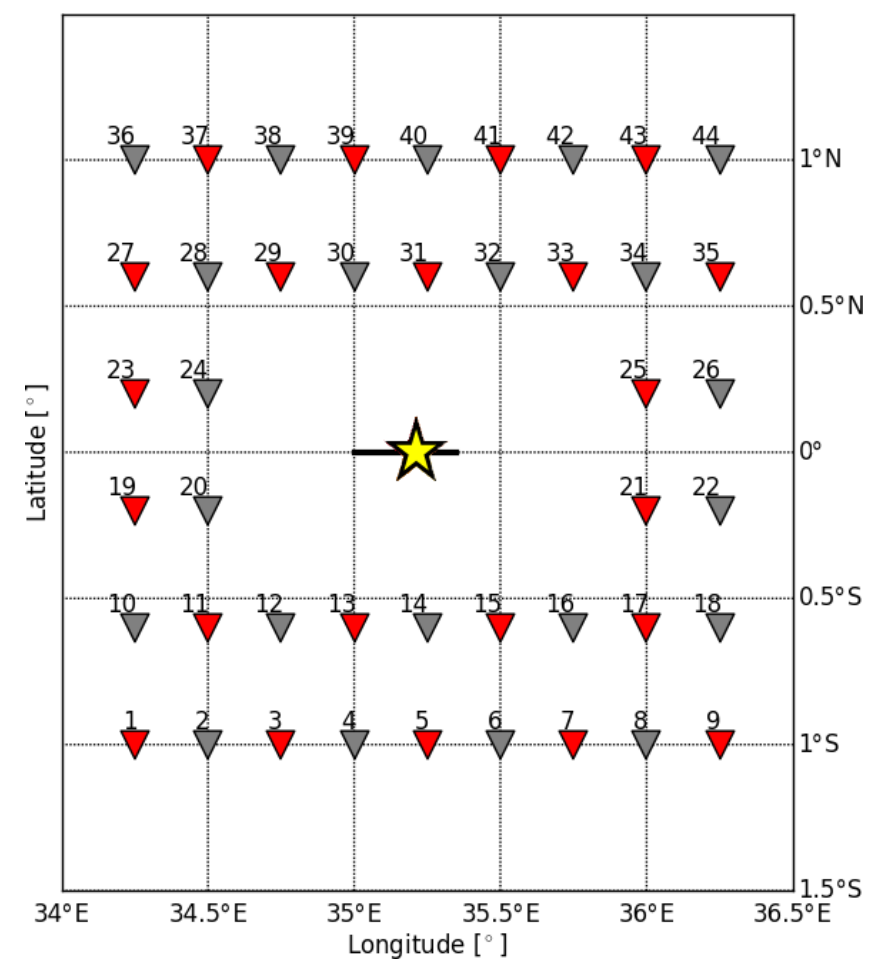

Figure 1. 44 receivers, marked as gray (only used without rotation data) and red triangles (used for all inversions), are set up around the source with a spacing of $0.4^{\circ}$ latitude and $0.25^{\circ}$ longitude. The fault trace is illustrated as a black line with constant latitude of $0^{\circ}$. The yellow star marks the epicenter of the earthquake.

point source seismograms in each subfault, appropriately delayed in time, with the aforementioned software Instaseis. This library has to be computed only once and contains the seismic wave field, $G_{k}^{r}$ corresponding to subfault $k$, station $r$. The second parameter is the rise time $(R)$, which is also homogeneous for the entire fault. It expresses the duration of the slip and is incorporated in the source time function used for the forward modeling. We choose the source time function to be an ordinary ramp function. The third parameter that contributes to the seismic response is the final slip amplitude. This parameter is defined for all subfaults individually.

The complete seismic response $v_{r}(\omega)$ in the frequency domain at station $r$ summed over all $N=24$ subfaults with circular frequency $\omega=2 \pi f$, the individual slip amplitude slip for each subfault, the rupture velocity $c_{\text {rup }}$, the source time function $S$ with the rise time $R$ and Green's function $G$, is expressed in Eq. (6).

Note that there are two parameters (i.e., rupture velocity and rise time) that are not linearly related to displacement or velocity, contrary to the 24 slip amplitudes. We therefore invert for 26 kinematic source parameters.

$v_{r}(\omega)=\sum_{k=1}^{N} \operatorname{slip}_{k} \cdot \exp \left[-i \omega t_{k}\left(c_{\text {rup }}\right)\right] \cdot G_{k}^{r}(\omega) \cdot S(R, \omega)$ 


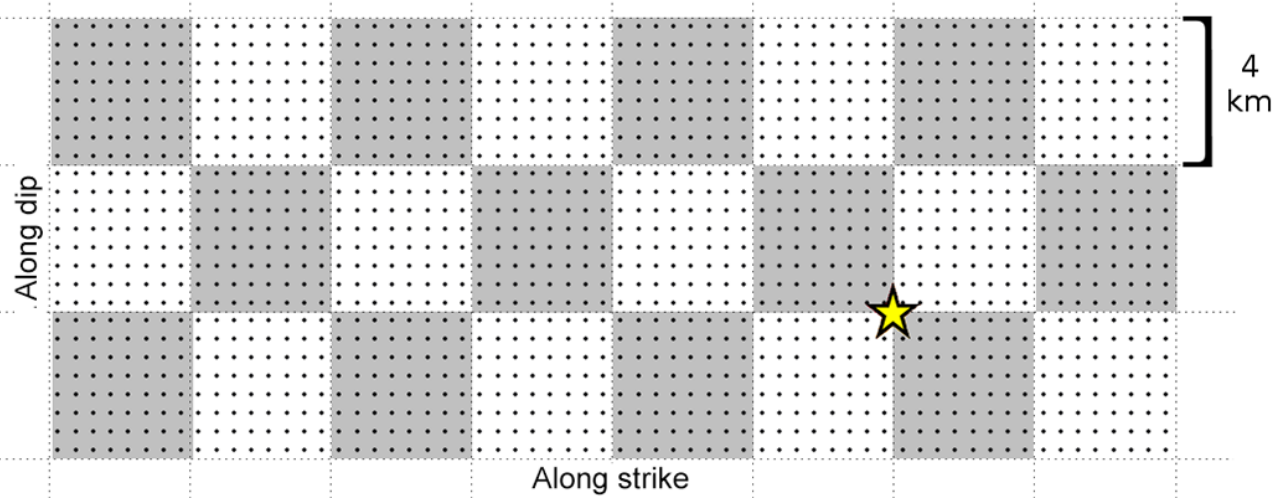

Figure 2. 2-D illustration of the fault neglecting constant third dimension. The fault is broken up into 24 subfaults, each consisting of 64 point sources. Each subfault will rupture appropriately delayed in time, depending on the distance to the hypocenter. The hypocenter (marked as a yellow star) is set to be exactly between four subfaults for easier computation of the rupture times of the subfaults.

The parametrization of the slip on the fault is illustrated in Fig. 3. We show heterogeneous slip amplitude as varying colors for each subfault, ranging from $0.6 \mathrm{~m}$ in all four corners up to $3.2 \mathrm{~m}$ in the eastern center part of the fault, i.e., one of the four subfaults closest to the hypocenter (yellow star). Because rupture velocity $\left(2700 \mathrm{~m} \mathrm{~s}^{-1}\right)$ and rise time $(0.8 \mathrm{~s})$ are homogeneous across the fault, it is sufficient to state the single values, which are valid for the entire fault. We add Gaussian noise to all synthetic seismograms to render our synthetic study more realistic. Because we calculate velocity and rotation rate seismograms individually, the noise on both data types is not correlated. We set the noise level to $10 \%$ of the maximum amplitude observed in all recordings for each respective data type for all scenarios. This ensures that a potential increase or decrease in inversion quality does not result from different noise levels.

We compare the inversions for two different fault mechanisms. For Scenario I we choose a pure left-lateral strikeslip event (rake parallel in strike direction). For Scenario II, its dip (angle between the fault and the horizontal plane) is changed from 90 to $45^{\circ}$ and the sliding now occurs perpendicular to strike direction (rake $\lambda=-90^{\circ}$ ). The fault shows pure dip-slip behavior and fractures also in vertical direction (Fig. 1).

\section{Inversion results}

For the first inversions in each scenario, we choose to use three-component velocity seismograms from all 44 stations. To reasonably compare the inversions with and without rotation rate data, we use only half of the stations (22) for the second inversion. With six-component (three velocity, three rotation rate) stations, the same number of seismograms (132) will be used for both inversions. The stations in the second inversions have been selected regularly (every second one) to achieve the lowest azimuthal gap possible.
Our third analysis consists of a statistical evaluation of 200 inversions, each with a randomly selected set of receivers, for both mechanisms. Since our previous results are achieved with a regular spacing of the receivers, we try to answer the question of whether the results are dependent on the spatial distribution of the receivers. Especially the different energy ratios of the rotation components suggest that there should be differences in the inversion results with varying station distribution.

We illustrate the results for both inversions for the 24 slip amplitudes in Scenario I with a regular station distribution in Fig. 4. Each panel represents a subfault with the same arrangement as in Fig. 2. In each panel the homogeneous prior pdf is drawn as a black dashed line and the true value for the slip in meters in each subfault is marked with a red line. The posterior pdf for the $3 \mathrm{C}$ inversion is drawn as a solid black line, the one for the $6 \mathrm{C}$ inversion in blue. We calculate the information gain for each parameter, i.e., for each subfault, individually. These values, in units of bit, are also shown in Fig. 4 in the corresponding colors (black/blue) for the $3 \mathrm{C} / 6 \mathrm{C}$ inversion. We see that the results clearly vary with depth. Not only does the cumulative information gain for the $3 \mathrm{C}$ and $6 \mathrm{C}$ inversion decrease the deeper the subfault is buried (top row: 4.86 bit (3C), 5.81 bit (6C); middle row: 4.47 bit (3C) 4.80 bit (6C); bottom row: 4.31 bit (3C), 4.37 bit (6C)), but also the percentage of how much the information gain increased in the $6 \mathrm{C}$ inversion compared to the $3 \mathrm{C}$ inversion decreases with increasing depth (Table 1). This indicates that shallower subfaults are resolved better in the inversion and rotation rates are likely to improve these subfaults more than deeper subfaults. It seems like the bottom row of the subfaults does not show any improvement by the incorporation of rotational ground motion data. This agrees with several studies (e.g., Bernauer et al., 2014; Cotton and Campillo, 1995; Semmane et al., 2005; Monelli and Mai, 2008; Mendoza and Hartzell, 1989). The total cumulative information gain over all 24 sub- 


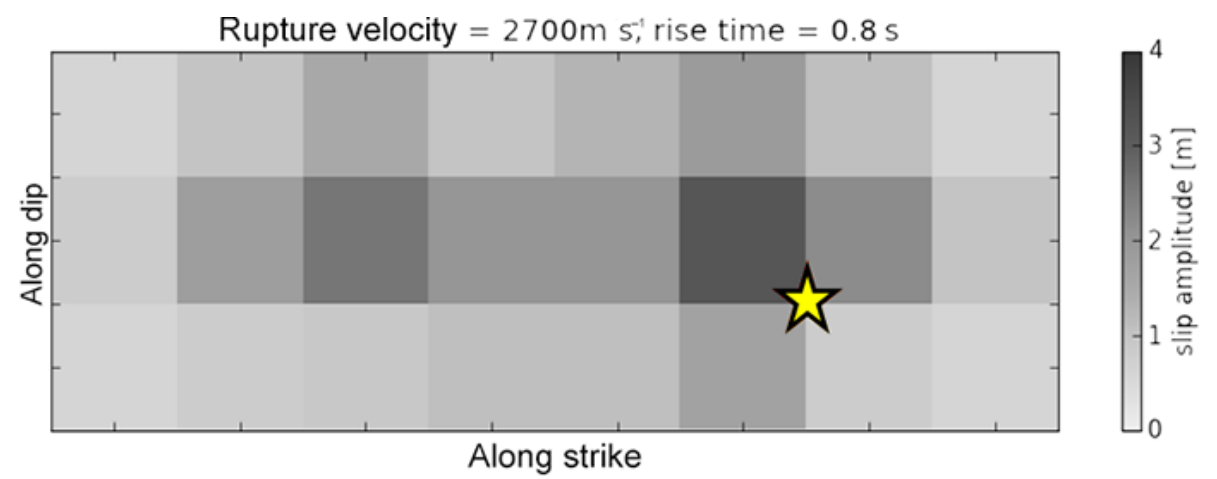

Figure 3. Illustration of the parameter space for the inversion. Rupture velocity and rise time are set to be homogeneous across the fault. There are 24 slip amplitudes, one for each subfault across the fault. The values range from $0.6 \mathrm{~m}$ in the corners to $3.2 \mathrm{~m}$ in one of the subfaults closest to the hypocenter (yellow star).

Table 1. Increase in information gain for $6 \mathrm{C}$ inversions compared to $3 \mathrm{C}$ inversions in percent. Subfaults are analyzed in the three horizontal layers (varying depth). The largest ratio of information gain increase for the slip amplitude between the $6 \mathrm{C}$ and the $3 \mathrm{C}$ inversion is in the deepest subfault layers. Rupture velocity and rise time benefit from rotation rate data for both types of scenarios, but the improvement is larger for the dip-slip scenario.

\begin{tabular}{lrr}
\hline Parameter & Strike-slip & Dip-slip \\
\hline Slip, overall (\%) & 9.86 & 32.13 \\
Slip, top row (\%) & 19.58 & 52.08 \\
Slip, middle row (\%) & 7.53 & 21.20 \\
Slip, bottom row (\%) & 1.32 & 20.75 \\
\hline Rupture velocity (\%) & 45.02 & 56.02 \\
Rise time (\%) & 17.77 & 28.47 \\
\hline
\end{tabular}

faults is with 14.98 bit about $9.86 \%$ larger for the $6 \mathrm{C}$ inversion than for the $3 \mathrm{C}$ inversion with 13.63 bit.

We illustrate the posterior pdf's for the two parameters that are not linearly related to displacement, i.e., rupture velocity and rise time, in Fig. 5. We use the same labeling as in Fig. 4. We cannot resolve the exact true values very well, but if one compares the information gain between the $3 \mathrm{C}$ inversion and the $6 \mathrm{C}$ inversion, there is a clear increase when incorporating rotation rate data into the inversion scheme. There is a $45.02 \%$ increase in information gain for inverting for rupture velocity and a $17.77 \%$ increase for inverting for rise time. This is again in agreement with the results from Bernauer et al. (2014), where the true values are also not captured and the increase in information gain is around the same order for both values. The recovery of the true values for these source parameters (rupture velocity and rise time) is a general problem when inverting for the kinematic source and strongly depends on the specific source-receiver geometry. Therefore, it is possible to have information gain on parameters through additional information or stations while not recovering the true parameter in terms of most-likely models.
In Fig. 6 we illustrate the results for a dip-slip fault in the same manner as Fig. 4. The decrease of inversion quality with depth is clearly visible again. A comparison between the cumulative information gain per row leads us to the assumption that slip amplitudes in the shallowest subfault layer seem well resolved, whereas the deeper layers tend to be poorly resolved in the inversion process (top row: 5.18 bit (3C), 7.87 bit (6C); middle row: 4.77 bit (3C) 5.78 bit (6C); bottom row: 4.50 bit (3C), 5.43 bit (6C)). We use these numbers to calculate the increase in information gain for the $6 \mathrm{C}$ inversion for each row (Table 1). The highest increase can be seen in the top row. The bottom row features only a slightly smaller increase than the row above. There is a much larger difference in the middle and the bottom row for a strike-slip fault. This information tells us that, for a normal fault, we are able to improve the inversion quality for the parameters of the deeper parts of the fault much better than in Scenario I by incorporating rotation ground motion data. The $6 \mathrm{C}$ inversion posterior pdf also seems to capture the true slip values quite well, especially on the sides and corners of the fault, compared to the results for a strike-slip fault. The total cumulative increase in information gain for all 24 subfaults is with a value of $32.13 \%$ more than 3 times higher than in Scenario I. The results for rupture velocity and rise time for a dip-slip fault are presented in Fig. 7. Although we see a clear improvement between 3C and 6C posterior pdf's, both parameters are not captured correctly by the inversions. There is, nevertheless, an improvement in resolving the true value with the $6 \mathrm{C}$ inversions for the rise time. For rupture velocity we do not see an improvement regarding the maximum of the posterior pdf.

The increase in information gain for rupture velocity $(56.02 \%)$ and for rise time $(28.47 \%)$ is also higher for Scenario II than for Scenario I.

The $6 \mathrm{C}$ inversions in the previous chapters are carried out with a manually predefined station selection.

The unequal distribution of energy ratios of rotation rate components might lead to the assumption that there is a 


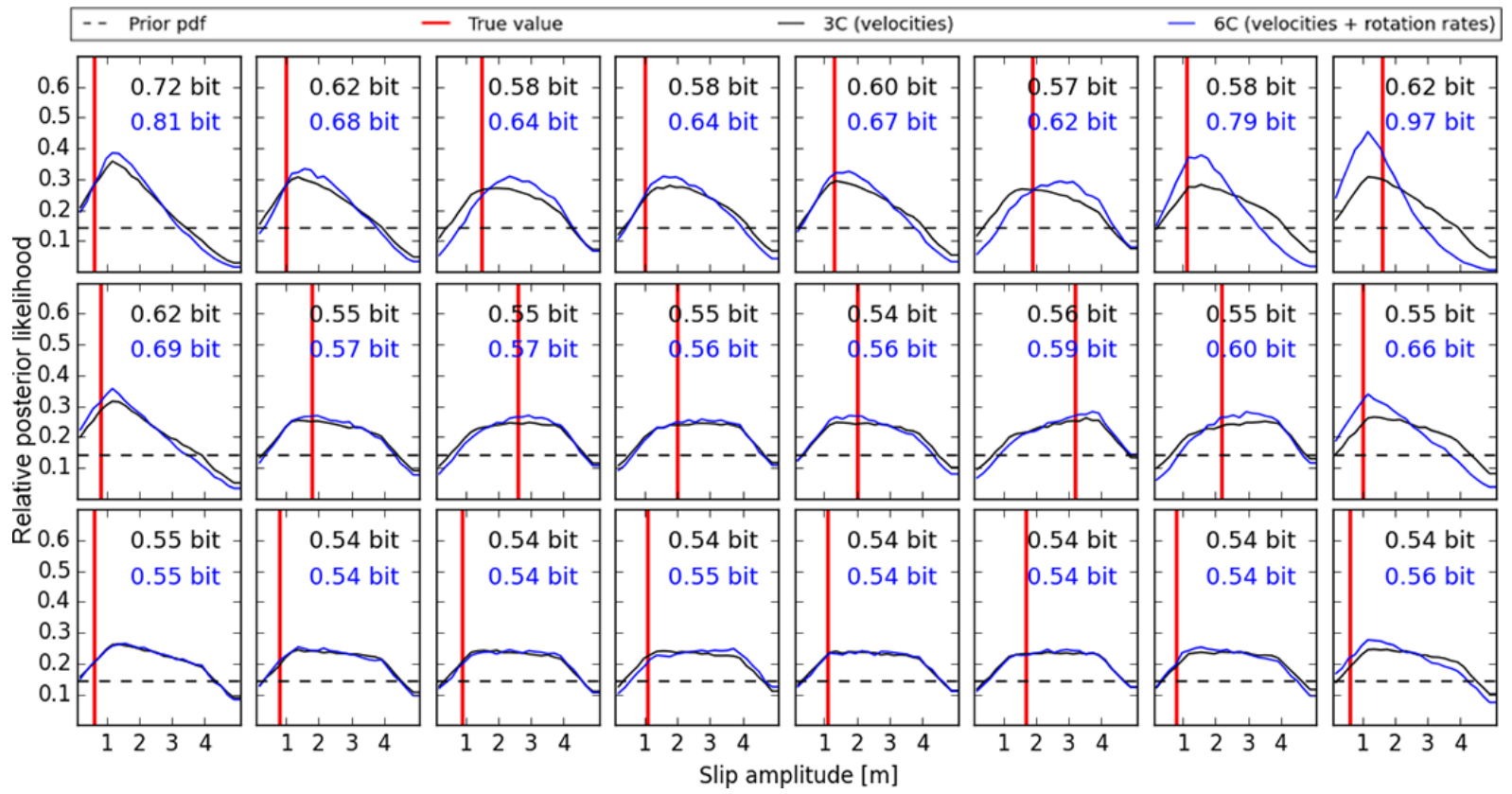

Figure 4. Inversion results for slip amplitudes for a strike-slip fault. Each panel represents a subfault with the same arrangement as in Fig. 2. For each subfault, the homogeneous prior pdf is drawn as a black dashed line, the true value for the slip amplitudes as a red vertical line, and the information gain is noted in the respective color of the inversion (3C in black and 6C in blue). The posterior pdf's for the 3C and 6C inversions are drawn as black and blue lines, respectively. The quality of the inversion shows a clear decreasing dependency with increasing depth. The bottom layer seems not to be able to be resolved better by the $6 \mathrm{C}$ inversion.
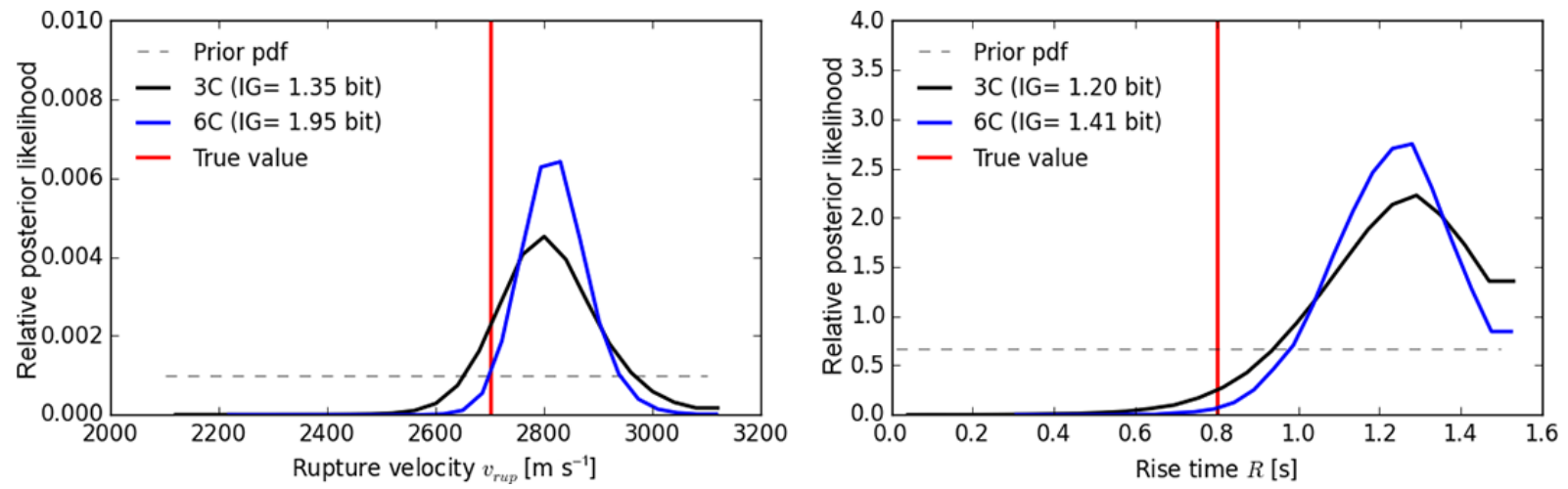

Figure 5. Inversion results for rupture velocity $v_{\text {rup }}$ (left) and rise time $R$ (right) for a strike-slip fault. The prior pdf is shown as a black dashed line, the true value as a vertical red line, and the posterior pdf for the $3 \mathrm{C}$ inversion as a black line and for the $6 \mathrm{C}$ inversion as a blue line. The information gain increased with incorporation of rotation rate data by $45.02 \%$ for inverting for rupture velocity and by $17.77 \%$ increase for rise time. The true values are not captured better by the $6 \mathrm{C}$ inversion.

higher information gain for the 6C inversion in Scenario II, because the regular receiver distribution leads to larger number of receivers lying north and south of the fault than east and west of the fault. These receivers feature a higher energy ratio of the horizontal rotation components. To minimize this factor we run $2006 \mathrm{C}$ inversions with 22 random stations each inversion, for both faulting types, and we show the results in Fig. 8 .

The results for a strike-slip fault are shown in the left column and for a dip-slip fault in the right column. We look on the slip for the top, middle, and bottom row individually to better assess the inversion quality dependence with depth for the different scenarios. Therefore there are five histograms for the information gain for the $2006 \mathrm{C}$ inversions. We mark the information gain value for the $3 \mathrm{C}$ inversion for each scenario with a red vertical line in the histogram plots. Slip (in gray), rupture velocity (in blue), and rise time (in green) are all colored differently to distinguish between the three types of parameters. 


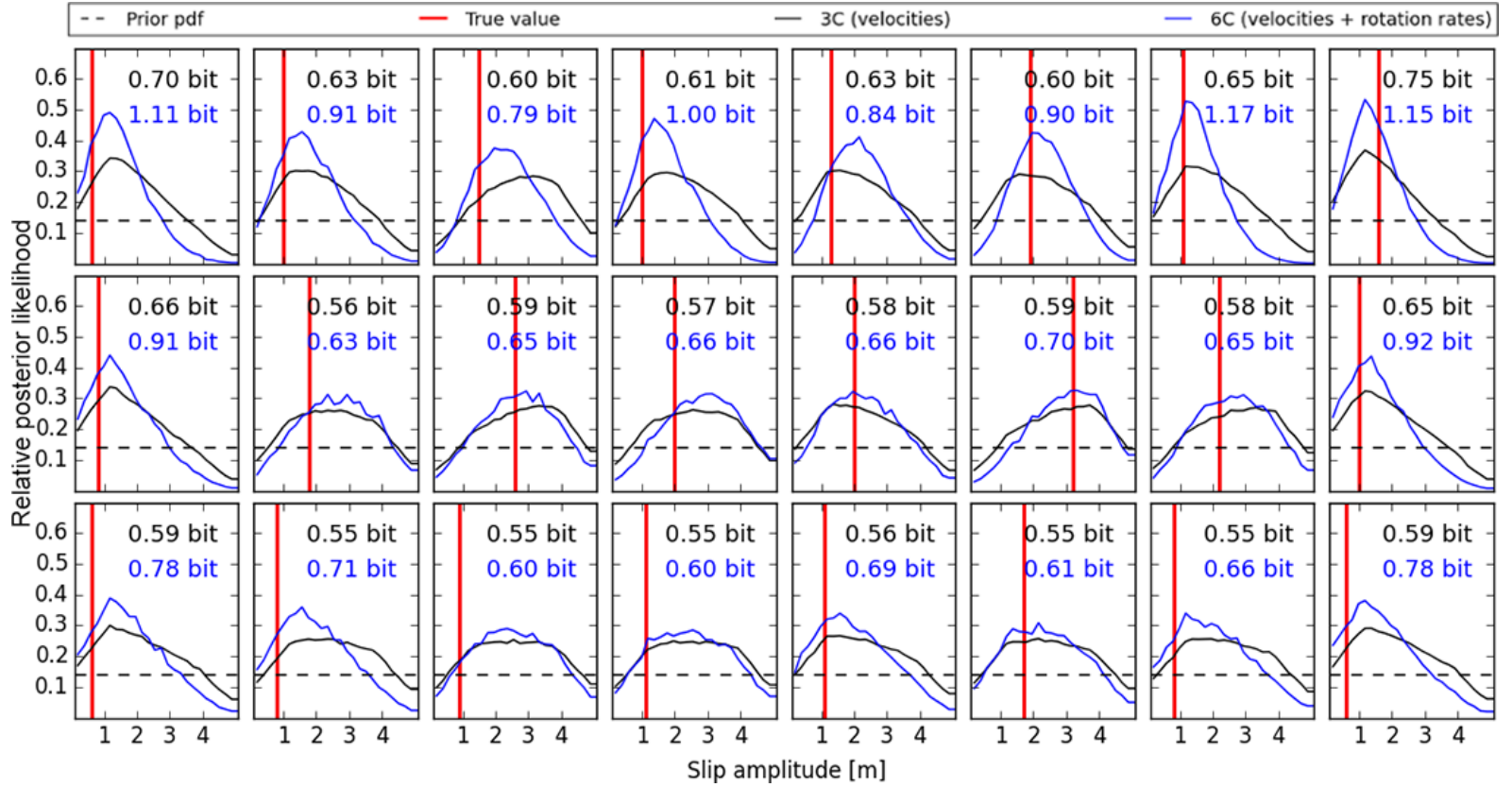

Figure 6. Inversion results for slip amplitudes for a dip-slip fault. Each panel represents a subfault with the same arrangement as in Fig. 2. For each subfault, the homogeneous prior pdf is drawn as a black dashed line, the true value for the slip amplitudes as a red vertical line, and the information gain is noted in the respective color of the inversion (3C in black and 6C in blue). The posterior pdf's for the $3 \mathrm{C}$ and $6 \mathrm{C}$ inversions are drawn as black and blue lines, respectively. The increase in information gain is higher compared to Fig. 4 for all subfaults but is still decreasing with depth.
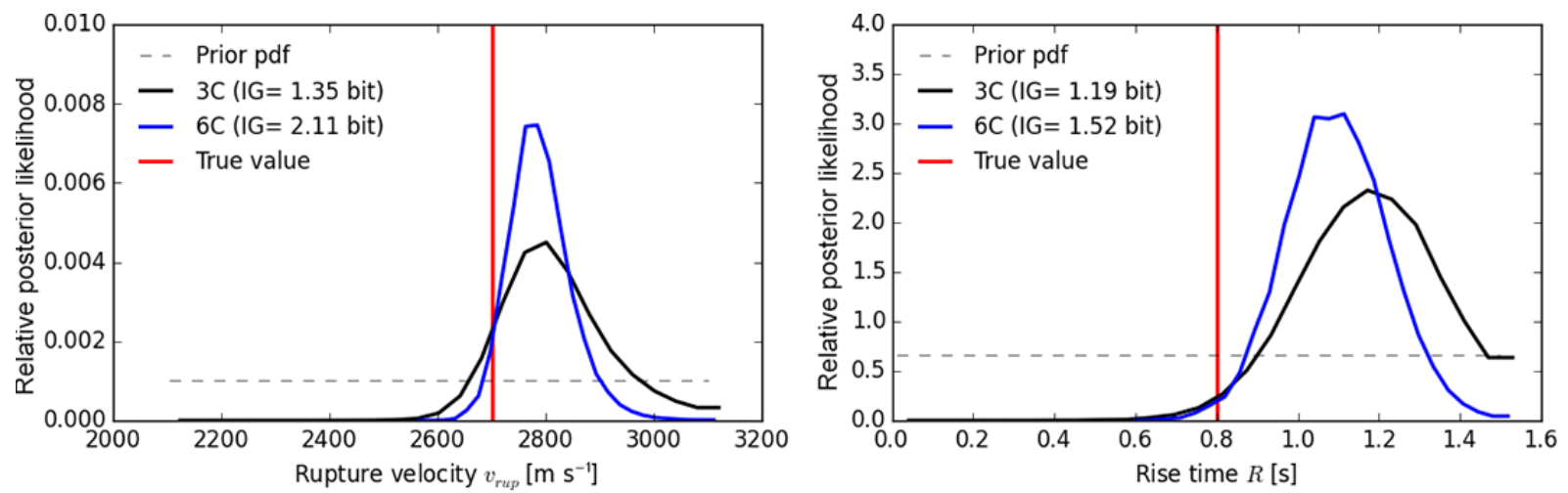

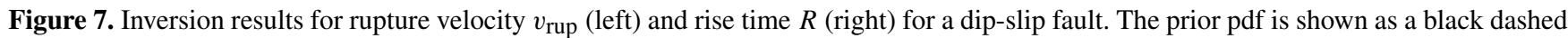
line, the true value as a vertical red line, and the posterior pdf for the $3 \mathrm{C}$ inversion as a black line and for the $6 \mathrm{C}$ inversion as a blue line. The information gain increased with incorporation of rotation rate data by $56.02 \%$ for inverting for rupture velocity and by $28.47 \%$ increase for rise time. Here, in contrast to Fig. 5, the true values are captured better by the $6 \mathrm{C}$ inversion than by the $3 \mathrm{C}$ inversion.

1. For the top layer of the subfaults, the information gain for a dip-slip fault (right) reaches the highest values, which is also the result from the previous inversion. There are some receiver selections that even increase the information gain compared to the results from the inversion with regular receiver spacing.

2. The middle subfault layer already features a smaller variance in the information gain distribution for both faulting types. We also see a decrease in information gain increase, compared to the top layer, which is to be expected from the results before. Additionally, there is a higher information gain increase for a dip-slip fault (right).

3. The deepest layer of subfaults shows the same information gain for $3 \mathrm{C}$ and $6 \mathrm{C}$ inversions for a strike-slip fault (left). The figure shows that including rotational ground motion does not contribute to improving the results for the slip on the bottom row. This is different for 

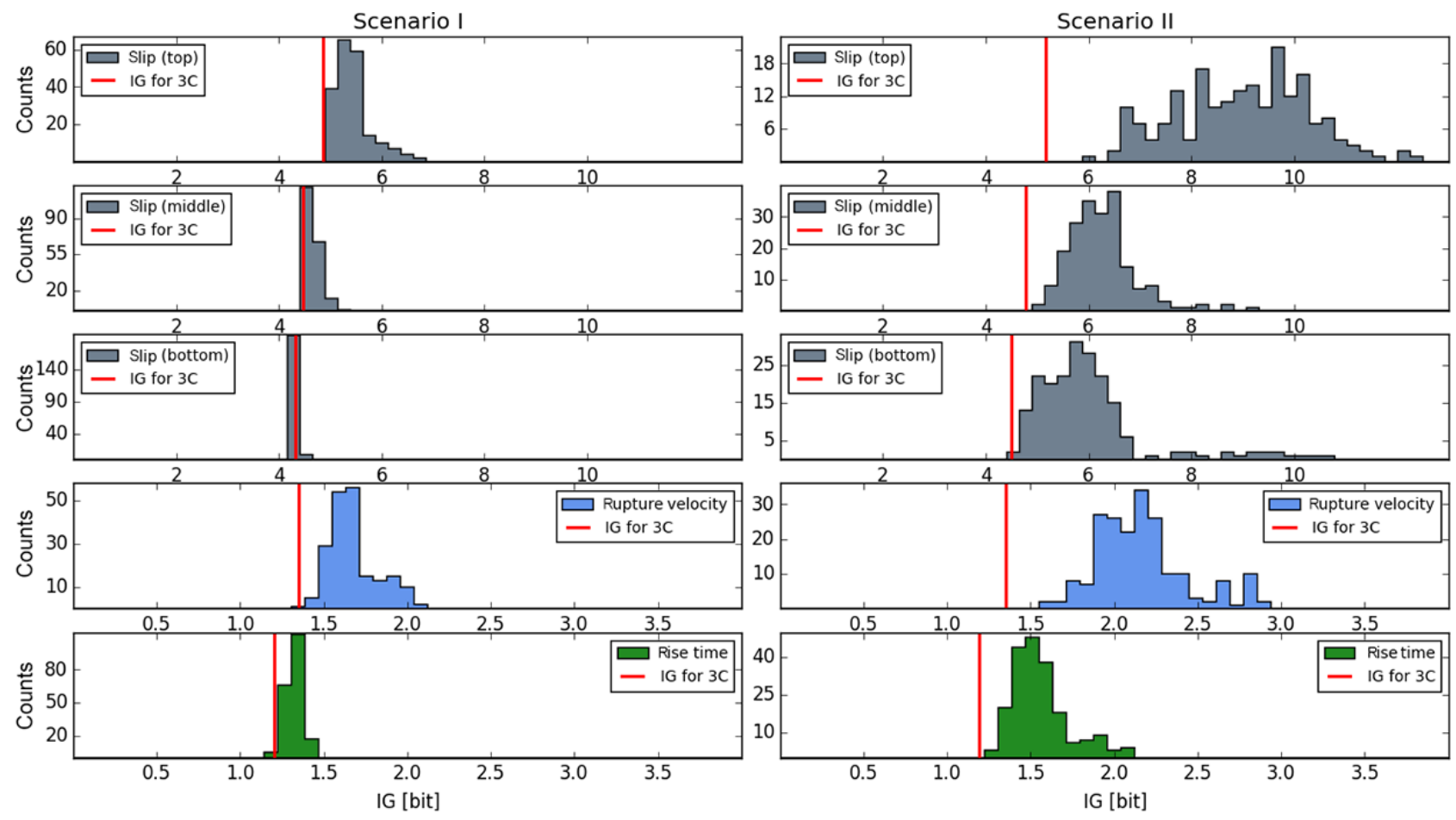

Figure 8. Histograms for the information gain for 200 inversions with random station distribution. The results for a strike-slip fault are shown in the left column and for a dip-slip fault in the right column. We add the information gain for the slip parameters row-wise to better assess the inversion quality dependence with depth. Slip (in gray), rupture velocity (in blue), and rise time (in green) are colored differently to distinguish between the three types of parameters. The statistical analysis validates the previous results. Independent of the choice of receivers (due to the random selection for each inversion) the information gain increase for the 6C inversion decreases with increasing depth; rupture velocity and rise time seem to benefit from the rotation rate data as well. Overall, the information gain increase is higher for Scenario II, even when randomizing the stations for the $6 \mathrm{C}$ inversion.

a dip-slip fault (right). Every inversion increases the information gain for slip amplitudes in the bottom layer. We see some inversions in which the information gain doubles compared to the $3 \mathrm{C}$ inversion. This major difference in the two scenarios is one of the key aspects of our results. The ability to invert for the slip amplitudes at higher depth is something that we only achieve with the incorporation of rotational ground motion rate data into the inversion. The statistical evaluation of 200 inversions with varying receiver consolidates this.

4. The inversions for rupture velocity result in a higher information gain for a dip-slip fault, even with random station distribution. Although inversions for rupture velocity for a strike-slip event (Scenario I) already benefit from rotation rate data, the improvement is even higher for a dip-slip (Scenario II) event.

5. The incorporation of rotation rate data only slightly improves the information gain for a strike-slip fault when inverting for rise time. The randomization of the receiver distribution decreases the information gain compared to the results from the inversion with a regular re- ceiver spacing ( $1.41 \mathrm{bit}$ ) for most of the inversions. The increase in information gain for a dip-slip fault is higher.

We compare the results for both types of faulting in Fig. 8 and see that for every row (all five parameters) the increase in information gain between $3 \mathrm{C}$ and $6 \mathrm{C}$ inversions is higher for a pure dip-slip event. The random selection of the receivers used in the inversions does not undo this result.

For the dip-slip mechanism, the inversion quality depends much more on the spatial distribution of stations (larger spreading in Fig. 8). This means that much more care needs to be taken when choosing locations with respect to the fault geometry for rotational sensors compared to translational sensors.

\section{Discussion}

With the range of calculated rotational ground motions between $10^{-7}$ and $10^{-4} \mathrm{rad} \mathrm{s}^{-1}$ we are able to draw practical conclusions from this synthetic study since effects in this range should be observable soon (Schreiber et al., 2009).

We expect to have higher energy in the rotational horizontal component signals for the dip-slip fault compared to the 


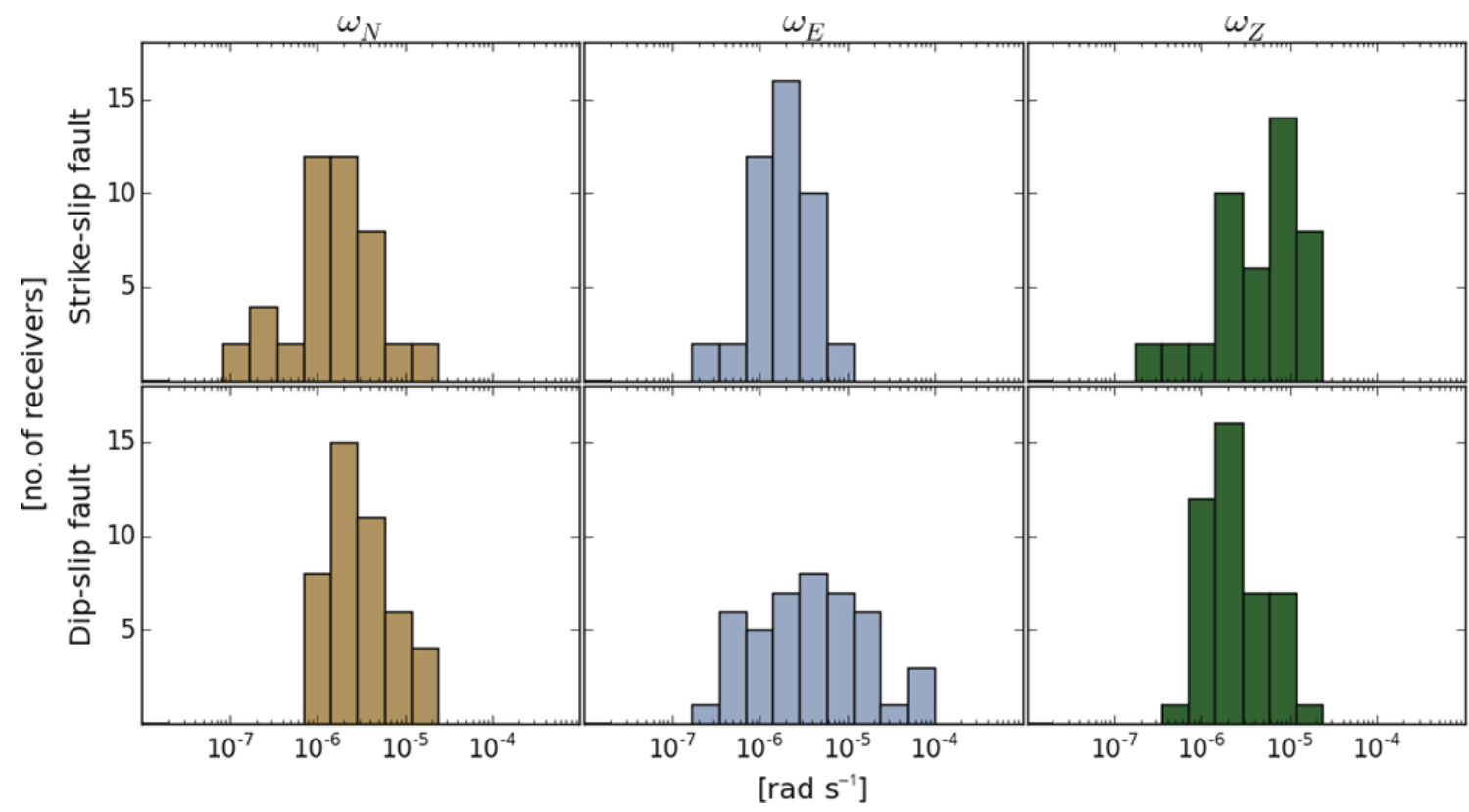

Figure 9. Histograms for maximum amplitudes of the three rotation components at all stations. The top row shows the distribution for the strike-slip fault, the bottom row for the dip-slip fault. The logarithmic scale for the rotation rates helps to get a sense for the amplitudes' order of magnitude. The histograms show how all 44 maximum amplitude values (for all 44 receivers) range between $10^{-7}$ and $10^{-4}$ rad s ${ }^{-1}$.

strike-slip fault. This would strengthen our assumption that this is a main reason for the increase in information gain for a fault that also slips in vertical direction. If we compute the total energy in all three rotational components measured at all stations, the energy of all rotation rate signals in the horizontal components makes up a very large part of the total energy measured at all stations for the dip-slip fault (57\% of the total energy), whereas the strike-slip fault features the highest energy ratio in the vertical component signals $(40 \%$ of the total energy for rotations around both horizontal axes), but horizontal rotation only contributes $20 \%$ of the total energy. This makes sense since we created our strike-slip earthquake to solely rupture in horizontal direction. To better assess the relevance of this study we check whether the theoretically predicted rotational motions could be observed in the field by state-of-the-art measurement devices. We therefore calculate the maximum amplitude for all 44 receivers for each of the three rotation rate components. They are shown in Fig. 9. The logarithmic scale for the rotation rates on the $x$ axes helps to get a sense for the amplitudes' order of magnitude. The distribution in each plot shows how all $44 \mathrm{am}$ plitude values (each for one receiver) range approximately between $10^{-7}$ and $10^{-4} \mathrm{rad} \mathrm{s}^{-1}$. Devices that are capable of measuring in this range of horizontal-component rotation rate amplitudes, e.g., Rotaphones, have been developed in recent years (Guattari, 2016; Brokešová and Málek, 2013, 2015). Fiber optic techniques that are able to measure in this range are currently under development (Velikoseltsev et al., 2012; Schreiber et al., 2009).
We show a summary of the improvement of the information gain between the different scenarios and parameters in percent in Table 1. Subfaults are analyzed by the three horizontal layers (varying depth). The largest difference in increase in information gain for the slip amplitudes is in the shallowest subfault layers. Rupture velocity and rise time benefit from rotation rate data for both types of scenarios, but the improvement is larger for the dip-slip event. The results for slip, rupture velocity, and rise time are also on the same order as the results in Bernauer et al. (2014) for a similar strike-slip event.

Rotation data seem to be most beneficial for inversions of kinematic parameters of sources that fracture at least partly in vertical direction. Additionally, the increase in information gain for rupture velocity and rise time suggests possible improvements in inversions for other seismological research areas. These parameters are important for rheology and friction laws used in dynamic rupture simulations (Tinti et al., 2009). Directivity measurements could also benefit from high-quality rupture velocity inversions (Somerville et al., 1997). Rotation rate data improved the inversions for the rise time as a parameter of the source time function. This helps when working with far-field ground displacements which are related to the derivative of the source time function.

A successful decrease in non-uniqueness in probabilistic finite-source inversion suggests manufacturing portable rotation sensors with the mentioned sensitivities. The noise level, which was set to be equal to the noise level for translational ground motions sensors of $10 \%$, should not exceed 
this to successfully improve inversion results (Bernauer et al., 2014).

\section{Conclusions}

Overall, non-uniqueness, which is an imminent problem in inversion theory, has been significantly reduced by the incorporation of synthetic rotational ground motion data.

The results from this study clearly suggest that seismological studies can benefit from the ability of seismometers to also measure rotational ground motions in addition to the conventional three translational components. This applies not only to finite-source inversions, which are a crucial part of seismological research, but probably to all work related to earthquake source inversions that relies on data recorded by seismic receivers.

We successfully showed how rotation rate measurements can improve the quality of seismological kinematic source inversions. This is most prominent for an earthquake which also fractures in vertical direction, due to the higher energy in the horizontal rotation components. It is very likely that the vertical displacement gradient, included in these components, leads to the observed decrease in non-uniqueness since this information can not be obtained with translation recordings on the Earth's surface.

\section{Data availability}

All data is freely available for download via the following link: https://drive.google.com/drive/folders/0B0_ LL6bBCLdSTmJfTTA5OUY3RVk?usp=sharing.

Acknowledgements. We would like to thank Lion Krischer for the support in professional code optimization. The authors acknowledge funding from the ERC advanced project ROMY (http://romy-erc.eu/). Further information necessary to replicate and build upon the reported research is available via correspondence by email.

Edited by: F. Romanelli

Reviewed by: J. Brokešová and W. Debski

\section{References}

Aki, K. and Richards, P.: Quantitative seismology, vol. 1, University Science Books, 2002.

Bernauer, F., Wassermann, J., and Igel, H.: Rotational sensors-a comparison of different sensor types, J. Seismol., 16, 595-602, 2012.

Bernauer, M., Fichtner, A., and Igel, H.: Reducing nonuniqueness in finite source inversion using rotational ground motions, J. Geophys. Res.-Sol. Ea., 119, 4860-4875, 2014.
Brokešová, J. and Málek, J.: Rotaphone, a Self-Calibrated SixDegree-of-Freedom Seismic Sensor and Its Strong-Motion Records, Seismol. Res. Lett., 84, 737-744, 2013.

Brokešová, J. and Málek, J.: Six-degree-of-freedom near-source seismic motions II: examples of real seismogram analysis and S-wave velocity retrieval, J. Seismol., 19, 511-539, 2015.

Cotton, F. and Campillo, M.: Frequency domain inversion of strong motions: application to the 1992 Landers earthquake, J. Geophys. Res.-Sol. Ea., 100, 3961-3975, 1995.

DeSalvo, R.: Review: accelerometer development for use in gravitational wave-detection interferometers, Bull. Seismol. Soc. Am., 99, 990-997, 2009.

Fichtner, A. and Igel, H.: Sensitivity densities for rotational groundmotion measurements, Bull. Seismol. Soc. Am., 99, 1302-1314, 2009.

Fichtner, A. and Tkalčić, H.: Insights into the kinematics of a volcanic caldera drop: Probabilistic finite-source inversion of the 1996 Bardarbunga, Iceland, earthquake, Earth Planet. Sc. Lett., 297, 607-615, 2010.

Guattari, F.: Blueseis-3A rotational seismometer, http://web.ixblue. com/cn/aw6ym/blueseis, last access: 15 April 2016.

Hastings, W.: Monte Carlo sampling methods using Markov chains and their applications, Biometrika, 57, 97-109, 1970.

Igel, H., Cochard, A., Wassermann, J., Flaws, A., Schreiber, U., Velikoseltsev, A., and Dinh, N.: Broad-band observations of earthquake-induced rotational ground motions, Geophys. J. Int., 168, 182-196, 2007.

Kennett, B., Engdahl, E., and Buland, R.: Constraints on seismic velocities in the Earth from traveltimes, Geophys. J. Int., 122, 108-124, 1995.

Lantz, B., Schofield, R., O'Reilly, B., Clark, D., and DeBra, D.: Review: Requirements for a ground rotation sensor to improve Advanced LIGO, Bull. Seismol. Soc. Am., 99, 980-989, 2009.

Mendoza, C. and Hartzell, S.: Slip distribution of the 19 September 1985 Michoacan, Mexico, earthquake: Near-source and teleseismic constraints, Bull. Seismol. Soc. Am., 79, 655-669, 1989.

Monelli, D. and Mai, P.: Bayesian inference of kinematic earthquake rupture parameters through fitting of strong motion data, Geophys. J. Int., 173, 220-232, 2008.

Monelli, D. and Mai, P.: Bayesian imaging of the 2000 Western Tottori (Japan) earthquake through fitting of strong motion and GPS data, Geophys. J. Int., 176, 135-150, 2009.

Mosegaard, K. and Tarantola, A.: Monte Carlo sampling of solutions to inverse problems, J. Geophys. Res.-Sol. Ea., 100, 1243112447, 1995.

Nigbor, R.: Six-degree-of-freedom ground-motion measurement, Bull. Seismol. Soc. Am., 84, 1665-1669, 1994.

Nissen-Meyer, T., van Driel, M., Stähler, S., Hosseini, K., Hempel, S., Auer, L., Colombi, A., and Fournier, A.: AxiSEM: broadband 3-D seismic wavefields in axisymmetric media, Solid Earth, 5, 425-445, doi:10.5194/se-5-425-2014, 2014.

Schreiber, K., Klügel, T., Velikoseltsev, A., Schlüter, W., Stedman, G., and Wells, J.-P.: The large ring laser G for continuous Earth rotation monitoring, Pure Appl. Geophys., 166, 14851498, 2009.

Semmane, F., Campillo, M., and Cotton, F.: Fault location and source process of the Boumerdes, Algeria, earthquake inferred from geodetic and strong motion data, Geophys. Res. Lett., 32, L01704, doi:10.1029/2004GL020664, 2005. 
Somerville, P., Smith, N., Graves, R., and Abrahamson, N.: Modification of empirical strong ground motion attenuation relations to include the amplitude and duration effects of rupture directivity, Seismol. Res. Lett., 68, 199-222, 1997.

Spudich, P., Steck, L., Hellweg, M., Fletcher, J., and Baker, L.: Transient stresses at Parkfield, California, produced by the M 7.4 Landers earthquake of June 28, 1992: Observations from the UPSAR dense seismograph array, J. Geophys. Res.-Sol. Ea., 100, 675-690, 1995.

Suryanto, W.: Rotational motions in seismology, theory and application, Ph.D. thesis, Ph. D. dissertation, Dep. of Earth and Environ. Sci. Geophys., Univ. of Munich, Munich, Germany, 2006.

Takeo, M.: Ground rotational motions recorded in near-source region of earthquakes, Geophys. Res. Lett., 25, 789-792, 1998.

Takeo, M. and Ito, H.: What can be learned from rotational motions excited by earthquakes?, Geophys. J. Int., 129, 319-329, 1997.

Tarantola, A.: Inverse problem theory and methods for model parameter estimation, Siam, 2005.
Tinti, E., Cocco, M., Fukuyama, E., and Piatanesi, A.: Dependence of slip weakening distance (Dc) on final slip during dynamic rupture of earthquakes, Geophys. J. Int., 177, 1205-1220, 2009.

Trifunac, M.: Earthquake Engineering, Non-linear Problems, in: Encyclopedia of Complexity and Systems Science, Springer, 2421-2438, 2009.

Twiss, R., Souter, B., and Unruh, J.: The effect of block rotations on the global seismic moment tensor and the patterns of seismic P and T axes, J. Geophys. Res.-Sol. Ea., 98, 645-674, 1993.

van Driel, M., Krischer, L., Stähler, S. C., Hosseini, K., and Nissen-Meyer, T.: Instaseis: instant global seismograms based on a broadband waveform database, Solid Earth, 6, 701-717, doi:10.5194/se-6-701-2015, 2015.

Velikoseltsev, A., Schreiber, K., Yankovsky, A., Wells, J.-P., Boronachin, A., and Tkachenko, A.: On the application of fiber optic gyroscopes for detection of seismic rotations, J. Seismol., 16, 623-637, 2012. 